\title{
Calibration for a hybrid MIMO near-field imaging system to mitigate antennas effects
}

\author{
Ha Hoang \\ Technological University Dublin, d16127394@mytudublin.ie \\ Zeeshan Ahmed \\ Technological University Dublin \\ Matthias John \\ Technological University Dublin
}

See next page for additional authors

Follow this and additional works at: https://arrow.tudublin.ie/ahfrccon

Part of the Computer Sciences Commons

\section{Recommended Citation}

H. Hoang, Z. Ahmed, M. John, P. McEvoy and M. Ammann, "Calibration for a Hybrid MIMO Near-field Imaging System to Mitigate Antennas Effects," 2020 International Workshop on Antenna Technology (iWAT), 2020, pp. 1-4, doi: 10.1109/iWAT48004.2020.1570608734.

This Conference Paper is brought to you for free and open access by the Antenna \& High Frequency Research Centre at ARROW@TU Dublin. It has been accepted for inclusion in Conference Papers by an authorized administrator of ARROW@TU Dublin. For more information, please contact arrow.admin@tudublin.ie, aisling.coyne@tudublin.ie,gerard.connolly@tudublin.ie. Funder: Science Foundation Ireland (SFI); European Regional Development Fund 
Authors

Ha Hoang, Zeeshan Ahmed, Matthias John, Patrick McEvoy, and Max Ammann

This conference paper is available at ARROW@TU Dublin: https://arrow.tudublin.ie/ahfrccon/49 


\title{
Calibration for a Hybrid MIMO Near-field Imaging System to Mitigate Antennas Effects
}

\author{
Ha Hoang ${ }^{*}$, Zeeshan Ahmed, Matthias John, Patrick McEvoy, Max Ammann \\ Antennas and High Frequency Research Centre \\ Technological University Dublin, Ireland \\ *d16127394@mytudublin.ie
}

\begin{abstract}
A calibration method for a high-resolution hybrid MIMO turntable radar imaging system is presented. A line of small metal balls is used in the calibration process to measure the position shift caused by undesired effects of the antennas. The unwanted effects in the near-field antenna response are analysed and significantly mitigated based on the referential features of the MIMO configuration.
\end{abstract}

Keywords-UWB radar system, near-field scattering imaging, inverse scattering problem, radar calibration, MIMO radar, antenna effects.

\section{INTRODUCTION}

Turntable radar imaging systems for high-resolution diagnostic imaging of complex objects are able to observe objects in arbitrary orientations and with a minimum of equipment [1]. The long scanning duration is a disadvantage of these systems. While multiple-input multiple-output (MIMO) systems can capture data about the object with only one snapshot in multiple views, a hybrid combination of turntable system and MIMO system can increase capture speed with little additional effort. Moreover, a differential feature in the MIMO channel responses can be utilized for system calibrations to mitigate the errors and/or system imperfections.

The calibration of a practical system plays a vital role in the improvement of reconstructed image accuracy. The accuracy of non-ideal electromagnetic acquirement systems is affected by many factors, in which near-field characteristics of antennas are significant. The imperfection of $\mathrm{S}_{11}$ characteristic of a bi-directional antenna and mutual near-field coupling between transmit-receive pairs of mono-directional antennas are factors degrading the system performance. To mitigate coupling effects in a MIMO system, a subtraction between the two measurements with and without the object were implemented in [2] and [3]. Another effect of the antennas in near-field response is the gain, amplitude and phase dependence on the direction of arrival (DoA). In [3] an adaptive weighting technique was proposed to calibrate these errors in a frequency domain process; however the method needs the measurement data at the exact position.

In this paper, a hybrid MIMO - turntable radar imaging system and a calibration method to reduce the effects of the antennas on the system performance with mitigation of complexity in measurement arrangement are reported.

\section{SySTEM AND ANTENNAS EFFECTS}

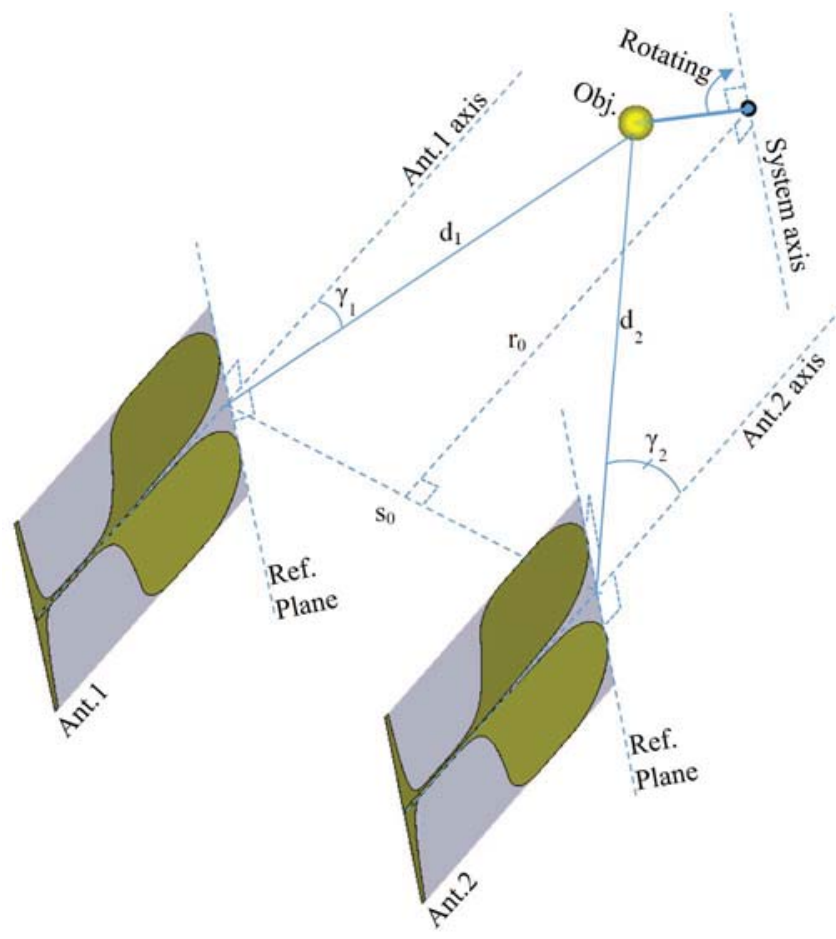

Fig. 1. System configuration.

Fig. 1 shows the configuration of the imaging system. It includes the two vertical-parallel fixed-mounted Vivaldi antennas (Ant.1 and Ant.2) with a spacing of $\mathrm{s}_{0}$. The two antenna axes and the system origin $\mathrm{O}$ are in the same plane (system plane), and the system axis is normal to the plane. The plane containing the system axis and the middle of $\mathrm{s}_{0}$ segment is parallel to the two antennas, and the distance from the system axis to the plane containing the two antennas reference planes is $r_{0}$. However, the two last conditions are not strict because the tolerances can be fixed by the calibration process. The object is placed on the turntable in the system plane, and it rotates around the system axis with the angular step of $\Delta \varphi$. The system parameters are shown in Table I.

TABLE I. SYSTEM PARAMETERS

\begin{tabular}{|c|c|}
\hline $\mathrm{s}_{0}$ & $180 \mathrm{~mm}$ \\
\hline $\mathrm{r}_{0}$ & $830 \mathrm{~mm}$ \\
\hline$\Delta \varphi$ & $1.5^{0}$ \\
\hline Rotating step number & 240 \\
\hline Antenna size $(\mathrm{L} \times \mathrm{W})$ & $130 \times 120 \mathrm{~mm}^{2}$ \\
\hline System Bandwidth & $10 \mathrm{MHz}-39.9 \mathrm{GHz}$ \\
\hline
\end{tabular}


In this MIMO system, each antenna plays the role of a transmitting antenna, a receiving antenna or both and a time division multiplexing scheme is used. Concurrently, one antenna is in transmitting and receiving mode while the other is only receiving. The two antennas have four combinations of channels (corresponding to four active virtual observed angles) for the measurement of scattering characteristic of the object at each position of the mechanical rotation. Furthermore, in practice, the speed of electrical measurement is faster than mechanical state change of the turntable. Thus, this hybrid MIMO configuration can increase density of observed directions in the system plane space and/or increase data acquirement speed compared to a single-channel system. The time-domain inverse scattering algorithm is applied to reconstruct the object scattering image from measurement data of the four channels of the two antennas combined with the rotating process [4], [5].

For an antenna operating concurrently in transmitting and receiving mode, the reflection of electromagnetic (EM) energy at the end edges is a major factor causing imperfection in $\mathrm{S}_{11}$ antenna characteristic, and it occults the small amount of received energy scattered from the object. Another negative effect is mutual near-field coupling between the two antennas. This can be considered as a near-field channel between one transmitting antenna and another receiving antenna through a small distance $\left(\mathrm{s}_{0}\right)$. This received EM energy proportion also masks the desired received signal scattered from the object.

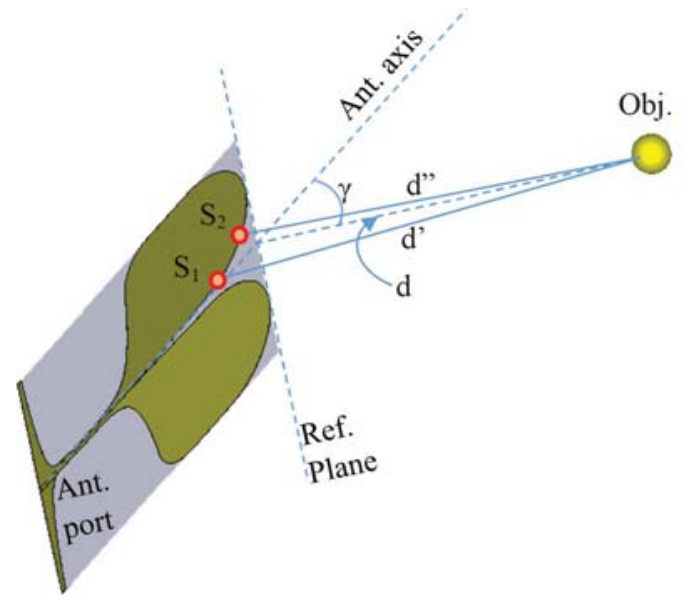

Fig. 2. DoA dependence effect in Vivaldi antenna response.

Fig. 2 shows another undesired effect of Vivaldi antennas depending on the direction of arrival (DoA). An example of ray-model propagation paths of scattered EM energy from the object to the antenna port starts from the object over path $d$ ' to a scattering point $\mathrm{S}_{1}$ on the Vivaldi edge and over the rest of the Vivaldi edge toward the antenna port when the antenna act as a receiver. The path is reversed in transmitting mode. Assuming that the distance $d$ to the object is not changed, when the arrival angle $\gamma$ increases, the sub-path in the air $d^{\prime}$ of the ray-model propagation path is decreased, and the integration of multipath along the edge has a corresponding decline. The far-field model shows that $d$ is constant versus $\gamma$. This DoA dependence effect leads to a significant error when the inverse scattering algorithm is applied to reconstruct the scattering image of the object space with the far-field model.

\section{CALIBRATION SCHEME AND RESUltS}

To mitigate the effects of the imperfection of $S_{11}$ characteristic and mutual coupling between the two antennas, the background subtraction method is applied. However, the slow ripple over the temperature in the response of the system transceiver can reduce the effectiveness of this method. The distance $r_{0}$ from the antenna reference planes to the system axis is chosen large enough that the systems intrinsic reflection and coupling signals arrive earlier than the scattered signals from the object. This can reduce the effect of slow ripple. The upper bound of $r_{0}$ depends on amplitude of the scattering object signals compared to the system noise level and the range of $\gamma$ angle. In this work, $r_{0}$ is chosen $830 \mathrm{~mm}$.

The spatial tolerance in the arrangement of system components significantly affects the system accuracy. An equivalent distance calculated from practical measured propagation time (from the antennas to the system origin) to calibrate for a tolerance of the nominal distances $r_{0}$ is necessary. This can be achieved with a planar reflector placed in the origin. To reduce the rigour in the measurement arrangement, the highly directional characteristic in radar cross-section of the plane is utilized in finding the exact angle of the reflecting plane on the turntable based on finding peak of the received signal which is transmitted from the other antenna. Fig. 3 shows the $230 \times 230 \mathrm{~mm}^{2}$ reflecting plane in the centre of the system turntable.

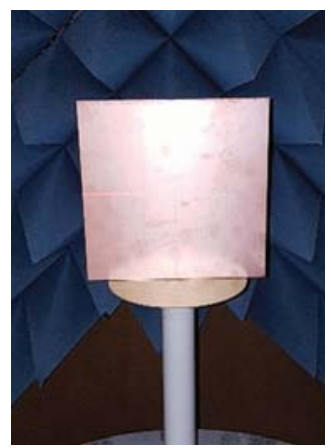

Fig. 3. Reflecting plane for system origin calibration.

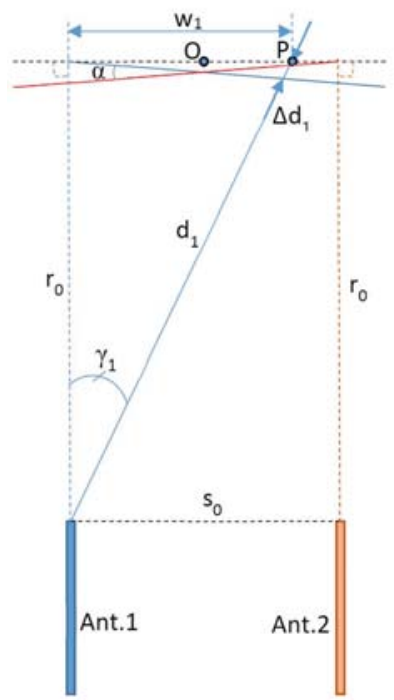

Fig. 4. Effect of the DoA dependence to a shift in object position measurement result. 
The effect of DoA dependence of the Vivaldi antennas to a shift in object position measurement result is explained in more detail in Fig. 4 . When the antenna 1 measures an object at $\mathrm{P}$, the measurement position is shifted by $\Delta \mathrm{d}_{1}$, which is a function of the angle $\gamma_{1}$. For objects at the points around $s_{0}$ region on the horizontal line at system origin $\mathrm{O}$, the shift $\Delta d_{l}$ can be approximated by a linear function versus the distance $w_{l}$ shown by the blue line in the Fig. 4 . Similarly, the red line shows the shift when measured by the antenna 2 and the differential angle between the two lines is $\alpha$. In general, the shift corresponding to $\gamma_{1}$ (or $\left.\gamma_{2}\right)$ at each point in the object space can be estimated and mitigated based on the angle $\alpha$. This shift also occurs in the reconstructed image when measured separately by antenna 1 and antenna 2 , and this is utilised for the calibration process.

The calibration sample for the DoA dependence consists of 7 steel balls of $11 \mathrm{~mm}$ diameter spaced linearly $40 \mathrm{~mm}$ apart close the horizontal line at the system origin. Exact positioning of this calibration sample is not necessary. Fig. 5 shows the sample and its placement in the system.

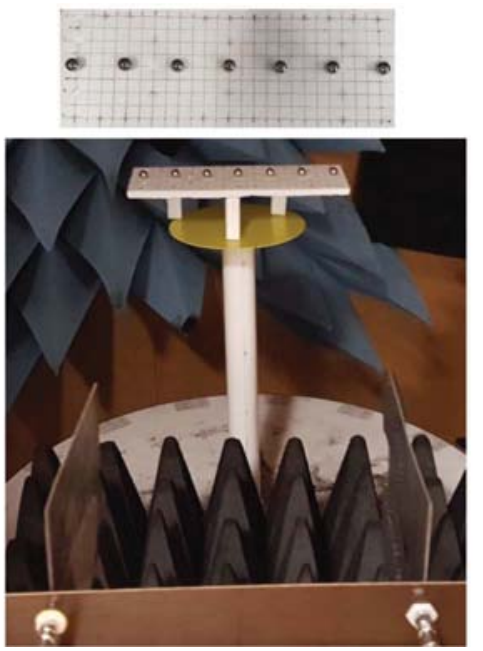

Fig. 5. (a) Calibration sample for DoA dependence and (b) the sample on the system turntable.

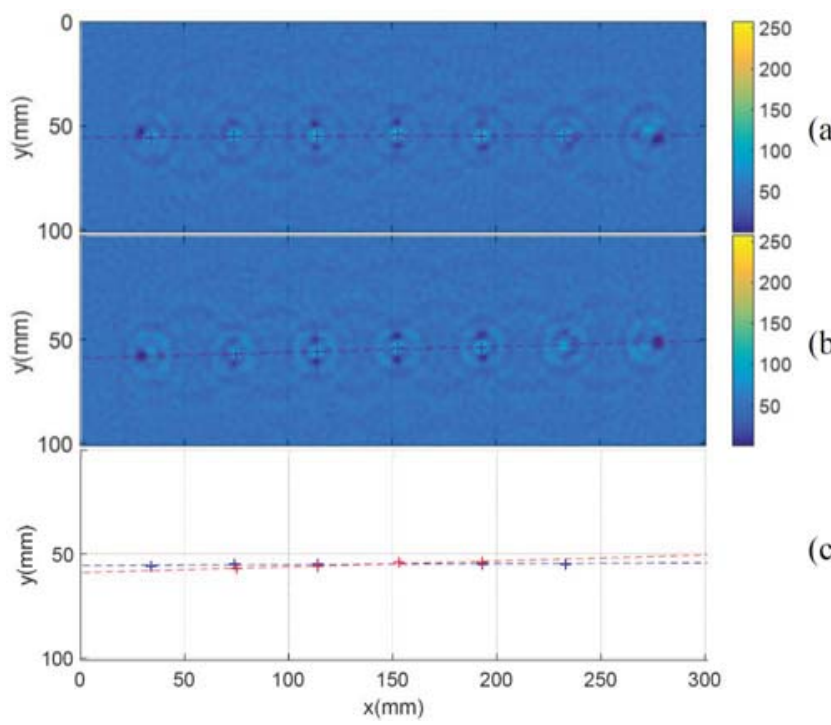

Fig. 6. Reconstructed images without DoA dependence calibration based on measurement channels of (a) ant.1 - ant.1, (b) ant.2 - ant.2 and (c) angle between the two estimated lines.
Fig. $6(a, b)$ shows the reconstructed images of the calibration sample based on measured data with the antenna 1 and 2 in monostatic mode. The shift affects the focus of the images at the ball positions, especially at the areas further from the system origin (image centre) where the peak values reduce.

The positions of the balls in the images are estimated based on the local peak finding algorithm, and these allow estimation of a line across the ball positions. The angle between the two lines can be calculated. In this case, the angle is $1.35^{0}$. The estimated elements are shown in Fig. 6 (c).

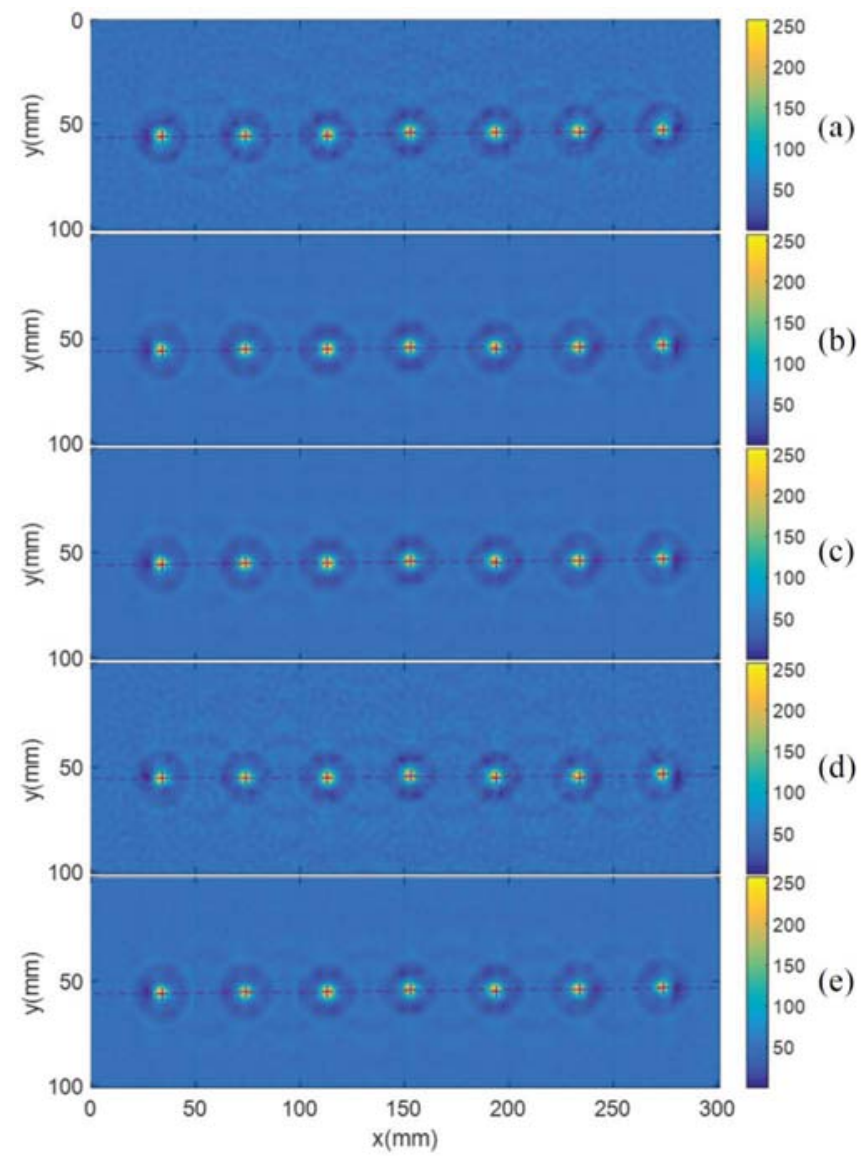

Fig. 7. Reconstructed images with DoA dependence calibration based on measurement channels of (a) ant.1 - ant.1, (b) ant.1—ant.2, (c) ant.2—ant.1, (d) ant.2-ant.2 and (e) all of four.

This angle value is a parameter for the inverse scattering algorithm to mitigate the DoA dependence effect of the antennas or the shifting $\Delta d_{1}$ and $\Delta d_{2}$ at each point in the object space. The results for the calibrated reconstruction of the calibration sample are shown in Fig. 7. The position shifting phenomenon of the object is reduced significantly, as is demonstrated by the improved focus as well as significant increase and regularity of the peaks in the images. This is also demonstrated in the reduction to $0.46^{\circ}$ in the estimated angle between the two lines corresponding to the measurement channels of antenna1 - antenna1 and antenna2 - antenna2.

When the calibration parameter has been estimated, the system can be used to measure other objects such as a sample with 31 steel balls of $11 \mathrm{~mm}$ diameter arranged into a shape of "TUD" characters. The sample and reconstructed scattering images are shown in Fig. 8 (a, b). The positions of the balls in the sample can be identified through the peaks in the scattering image with high accuracy, while the image without DoA 
dependence calibration in Fig. 8 (c) is blurred and the positions of the balls cannot be identified exactly.

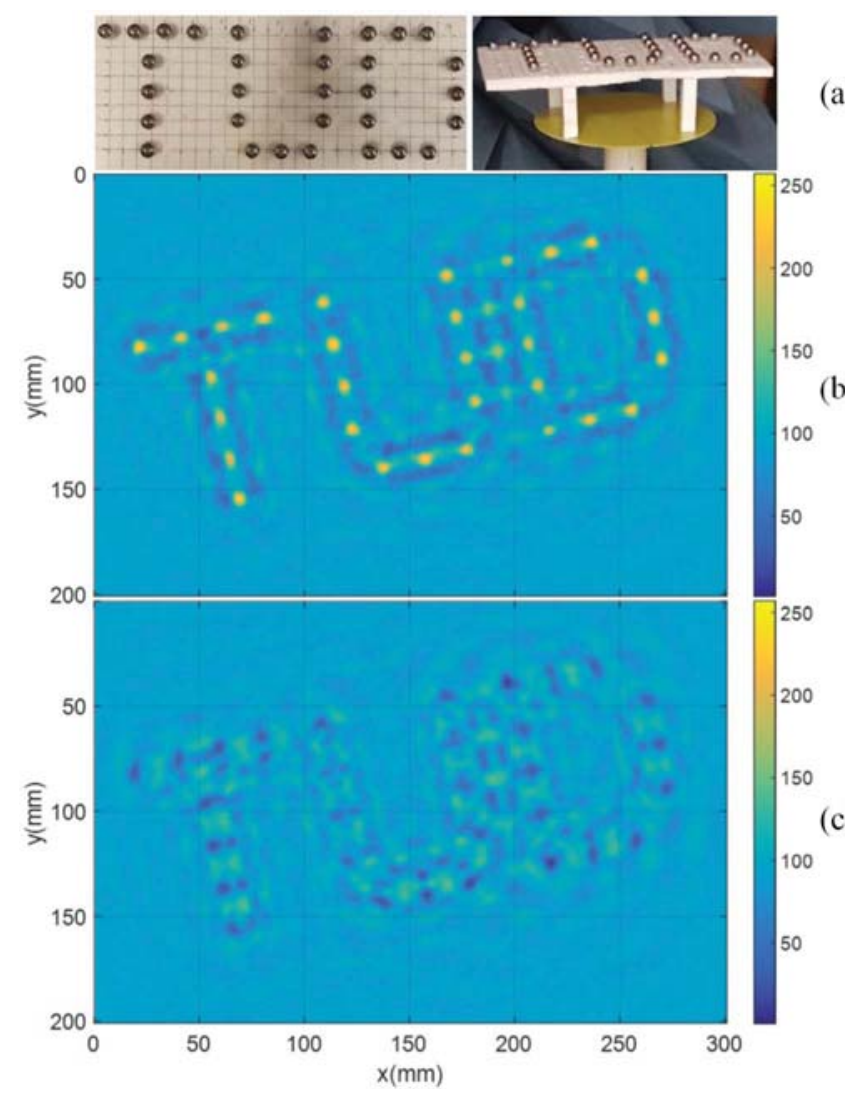

Fig. 8. Testing the system with (a) the "TUD" sample and the results (b) with DoA dependence calibration and (c) without DoA dependence calibration based on all four combination channels.

(b)

\section{CONCLUSION}

This paper presented a hybrid MIMO imaging system with undesired antenna near-field effects and demonstrated the advantage of a differential feature of the MIMO configuration for calibration. This feature was utilized in the calibration scheme with the relaxation in measurement arrangements. The effectiveness of this method in significant mitigation of the effects of the antennas was demonstrated in the improved focus of the reconstructed scattering images.

\section{ACKNOWLEDGMENT}

This publication has emanated from research conducted with the financial support of Science Foundation Ireland (SFI) and is co-funded under the European Regional Development Fund under Grant Number 13/RC/2077.

\section{REFERENCES}

[1] T. Vaupel ; T.F. Eibert, "Comparison and Application of Near-Field ISAR Imaging Techniques for Far-Field Radar Cross Section Determination", IEEE Transactions on Antennas and Propagation, vol. 54, p. $144-151,2006$.

[2] Georg Körner ; Daniel Oppelt ; Julian Adametz ; Martin Vossiek, "Novel Passive Calibration Method for Fully Polarimetric Near Field MIMO Imaging Radars", in Proc. 12th German Microwave Conference (GeMiC), Stuttgart, Germany, 2019.

[3] Yongze Liu ; Xiaojian Xu ; Guangyao Xu , "MIMO Radar Calibration and Imagery for Near-Field Scattering Diagnosis", IEEE Transactions on Aerospace and Electronic Systems, vol. 54, p. 442 - 452, 2018.

[4] Manh Ha Hoang ; Hong Phuong Phan ; Quoc Huy Dien ; Duc Lam Nguyen, "Design and experimental study of an ultra-wideband radar system", in Proc. International Conference on Advanced Technologies for Communications (ATC 2014), Hanoi, Vietnam 2014.

[5] A. Broquetas ; J. Palau ; L. Jofre ; A. Cardama, "Spherical wave nearfield imaging and radar cross-section measurement", IEEE Transactions on Antennas and Propagation, vol 46, p. 730 - 735, 1998 